\title{
Laminar Neuropathology in Alzheimer's Disease by a Modified Gallyas Impregnation
}

\author{
H. Heinsen, H. Beckmann, Y.L.Heinsen, F. Gallyas, S. Haas, and G. Scharff
}

The allocortex of the temporal lobe, consisting of the hippocampal formation, the subicular complex, and the entorhinal region, has been reported to be a site of predilection in Alzheimer's presenile changes as well as in senile dementia of the Alzheimer type (SDAT). Quantitative investigations in Bodian-stained tissue have been carried out by Kemper (1978), with the data summarized in a schematic drawing. The Bodian stain is not optimal for quantitative investigations since normal nerve cell processes are likewise impregnated. The Gallyas silver impregnation (Gallyas, 1971), which impregnates nearly selectively Alzheimer fibrillary changes, neuritic plaques as well as neuropil threads and argyrophilic grains (Braak et al., 1986; Break and Break, 1987) in afflicted nerve cell processes, appears to be a simpler and more reliable method which also works in 600 micrometer thick frozen sections.

Case Report. The brain was from a 58-year-old male with symptoms beginning 5 years before death. During an admission to a psychiatric state hospital 3 months before death, the patient was completely apraxic, agnostic, and aphasic with one generalized convulsion. The clinical diagnosis was Morbus Alzheimer; the cause of death was central circulatory failure. The left hemisphere was removed 6 hours post-mortem, formalin fixed ( $4 \%$ final concentration) for 3 months, treated with cryoprotective agents ( $30 \%$ saccharose), gelatine embedded, frozen in liquid isopentane at $-60^{\circ} \mathrm{C}$, and serially sectioned on a Tetrander with section thickness of 600 micrometers. At variance with Gallyas' (1971) original prescription, the frozen sections were pretreated in hot $\left(65^{\circ} \mathrm{C}\right)$ pyridine for 3 days, and a slightly modified physical developer was applied at $4^{\circ} \mathrm{C}$. Sections were alternately stained with a Nissl stain (gallocyanine) and impregnated according to Gallyas.

By virtue of the thick frozen sections, the topographic and laminar distribution of neuronal Alzheimer fibrils, neuritic plaques and nerve cell processes in the temporal allocortex can be macroscopically assessed (Fig. la). The hilus (Hil) exhibits a diffuse distribution of few nerve cells and some larger irregular neuritic plaques. The pyramidal cell layer from sector CA3 through CAl is stained with increasing intensity with the nerve cells of CA3 partly obscured by an argyrophilic fiber tract coursing medio-laterally in the stratum oriens. An additional band-like fiber tract which runs in a predominant rostro-caudal direction can be distinguished in the middle third of the hippocampal molecular layer (Fig. 1b, arrow). At the border of the lateral CAI sector-subicular complex (Fig. 1, labeled Sub) the pyramidal cells of the latter are obscured by three dark globular clouds mainly consisting of a mass of

Reprint requests to: Helmut Heinsen, M.D., Dept. of Psychiatry, Univ. of Würzburg, D-8700 Würzburg, FRG. 
Fig. 1. Section through hippocampus in patient with Alzheimer's dementia
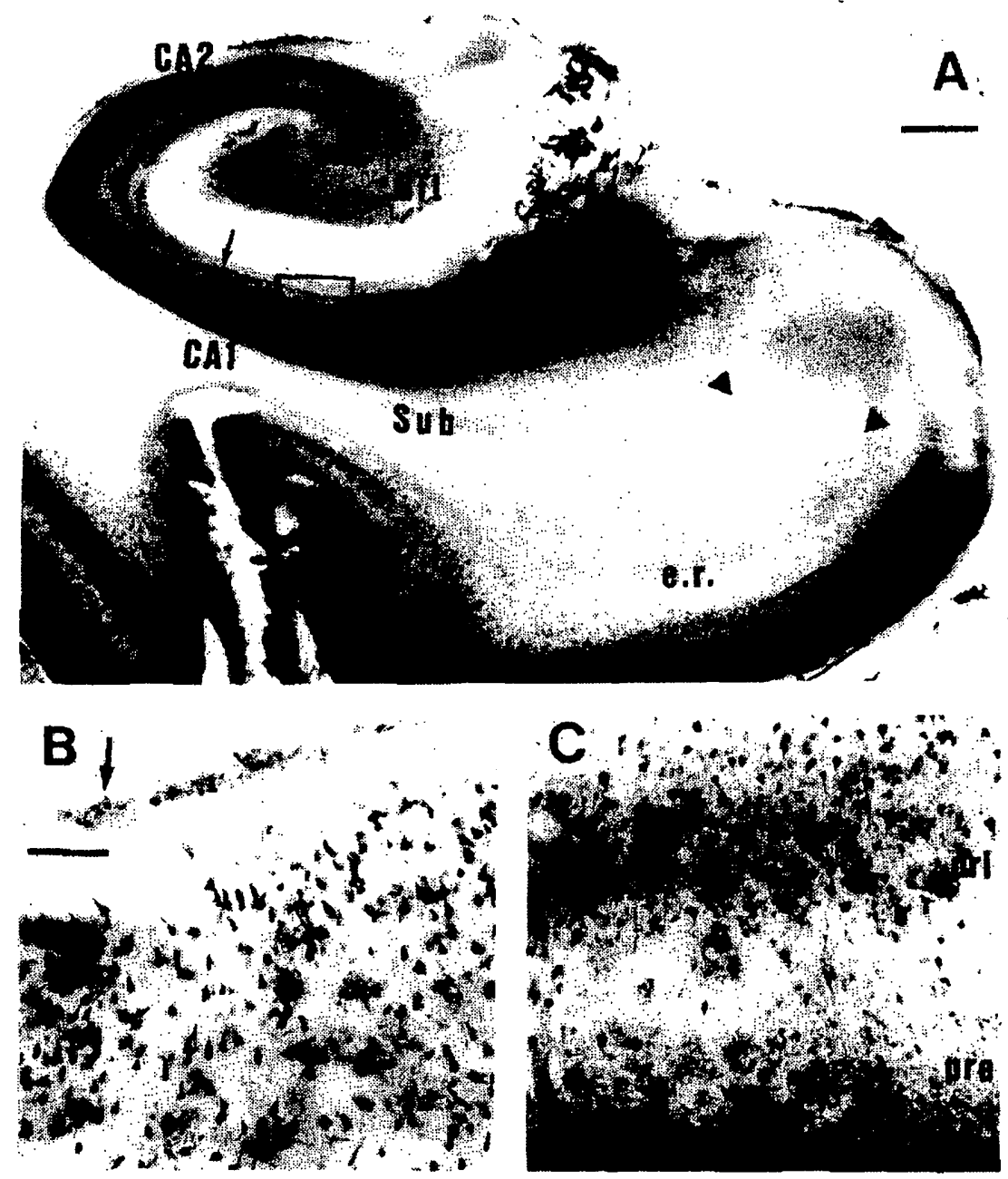

Top (1): Frontal section through left hippocampal formation and entorhinal region (e.r.) of the parahippocampal gyrus, about $5.4 \mathrm{~mm}$ rostral to the lateral geniculate body. Silver staining according to Gallyas (1971). Scale bar represents 1 $\mathrm{mm}$. Bottom left (2): Field CA1 from the hippocampal formation (upper square in (1)) in higher magnification. Bar represents $0.1 \mathrm{~mm}$. Bottom right (3): Entorhinal region (lower square in (t)), with same scale as bottom left. 
neuritic plaques. A field connecting the entorhinal region (Fig. 1 e.r., arrowheads) with the subicular complex is rather free from argyrophilic material with small zones of transition between the heavily afflicted subicular complex and the e.r. The superficial layers consisting of the lima zonalis and the layers pre-alpha through gamma (Fig. Ic, pre) and the deep pri-layers (Fig. lc, pri) of the entorhinal region are favorite sites of fibrillary changes and neuritic plaque accumulation (Fig. Ic). The intensely stained opaque superficial layers of the lamina molecularis and pre-alpha layer consist of numerous afflicted neuronal processes and neuritic plaques. In lowpower magnification the layers appear artifactually over-stained (Fig. 1), but in higher magnification staining, intensity is due to numerous neuropil threads and plaques.

We think that our modification consisting of serial thick frozen sections and silver impregnation with Gallyas' method is a fast and reliable procedure which complements previous findings and gives new insights into topographical as well as laminar distribution of pathologic changes in Alzheimer's disease, SDAT, and normal aging.

\section{References}

Braak, H., and Braak, E. Argyrophilic grains: Characteristic pathology of cerebral cortex in cases of adult onset dementia without Alzheimer changes. Neuroscience Letters, 76:124, 1987.

Braak, H.; Braak, E.; Grundke-Iqbal, I.; and Iqbal, K. Occurrence of neuropile threads in the senile human brain and in Alzheimer's disease: A third location of paired helical filaments outside neurofibrillary tangles and neuritic plaques. Neuroscience Letters, 65:351, 1986.

Gallyas, F. Silver staining of Alzheimer's neurofibrillary changes by means of physical development. Acta Morphologica Academiae Scientarum Hungaricae, 19:1, 1971.

Kemper, T. Senile dementia: A focal disease in the temporal lobe. In: Nandy, K., ed. Senile Dementia: A Biomedical Approach. Amsterdam: Elsevier/North-Holland Biomedical Press, 1978. p. 105. 